\title{
SHOULDER POSTURAL FATIGUE AND DISCOMFORT
}

\author{
A Preliminary Finding of No Relationship with Isometric Strength Capability \\ in a Light-Weight Manual Assembly Task
}

\author{
Steven F. Wiker *, Don B. Chaffin and Gary D. Langolf \\ Center for Ergonomics, University of Michigan, Ann Arbor, MI 48109 (U.S.A.)
}

(Received September 12, 1988; accepted in revised form May 22, 1989)

\begin{abstract}
Does greater strength capacity in the shoulder-complex afford increased protection against regionalized fatigue and discomfort induced by sustained awkward arm postures in light-weight manual assembly environments? This question was addressed by testing the relationship between differences in shoulder complex strength capacity, produced by variations in arm posture within a subject, and among subjects assuming equivalent arm postures, and severity of fatigue and discomfort sensed during a low-exertion manual performance task. Experimental findings showed that: (a) awkward arm postures produced substantial and rapid onset of postural fatigue and discomfort during a light-weight manual performance task where strength demands were low (i.e., less than 15 percent of maximum voluntary contraction $(M V C)),(b)$ variations in strength capability found among arm postures within an individual subject, or among subjects assuming the same arm posture, did not affect onset of substantial fatigue or discomfort when hands are postured near or above shoulder level, and (c) postures which simply appeared to be awkward, or which compromised strength capacity (e.g., working with the arm to the side of the body, or aligned in the coronal plane), did not necessarily increase discomfort of fatigue. Our findings suggest caution against sole reliance upon population or individual worker upper-extremity strength capabilities as predictors of fatigue and discomfort in the shoulder complex when manual exertions are small (e.g., light-weight manual assembly activities involving small parts or small hand-tools) and hands are postured at or above shoulder level.
\end{abstract}

\section{RELEVANCE TO INDUSTRY}

Stronger workers are often selected for manual assembly averhead, or when other awkward upper extremity postures must be sustained. Measurements of subject upper-extremity isometric strength capability in an array of arm postures, and computation of external shoulder load moments, were of no significant value in predicting or gauging onset or severity of fatigue and discomfort experienced in elevated arm postures. Ergonomists are encouraged to eliminate overhead work even in light-weight manual assembly environments and to extend their upper-extremity posture analyses beyond evaluation of

\footnotetext{
* Current address: Department of Industrial Engineering, University of Wisconsin, 1513 University Avenue, Madison, WI 53706, U.S.A. 
anthropometrics, shoulder external load moments, and upper extremity strength capability when specifying arm and shoulder postures in light-weight manual assembly environments.

\section{KEYWORDS}

Shoulder, posture, strength, fatigue, workplace, design.

\section{INTRODUCTION}

Numerous studies may be cited in which exertion magnitude is related to exertion endurance, and to onset and severity of sensations of localized fatigue and discomfort. Perhaps the most frequently cited are studies by: (a) Rhomert (1973), who recommends for avoidance of fatigue that exertions not exceed 15 percent of maximum voluntary contraction (MVC) capacity, and (b) Bjorksten and Jonsson (1977) who recommend limits of 15 and 8 percent of MVC for dynamic and static exertions, respectively. Though these guidelines are rarely violated in manual assembly operations involving small light-weight parts or hand-tools, it is not unusual to find worker complaining of postural discomfort and of localized muscle fatigue.

Ergonomists generally agree with the concept of minimizing job strength demands; especially if sustained and forceful excrtions are required. However, the utility of strength capability alone as a guide for specifying acceptable workplace postures, when task exertions are small, is uncertain. Factors beyond that of postural exertion demand, may also affect circulatory feed to, and mechanical strain in, musculoskeletal tissues. For example, working with hands and arms postured above the level of the heart, or at the limits of range of motion, might produce significant levels of fatigue and discomfort even when relative strength demands are quite small. If so, variations in strength capability found among upper extremity postures within individual workers, or between workers, may fail to account for posturally-based onset and magnitude of localized muscle fatigue (LMF) or discomfort.

The principal question addressed by this paper is whether shoulder strength capacity is a useful criterion in choosing among candidate upper ex- tremity postures when task exertions are small (e.g., use of sustained overhead postures while performing light-weight manual assembly operations). The experiment described in this paper was designed to test the relationship between measured shoulder strength within and among test subjects, and magnitudes of both global and regional fatigue and discomfort sensed during performance of a low-exertion manual performance task.

\section{BACKGROUND}

\section{Upper extremity circulation and arm elevation}

Studies of circulation in clevated arms have consistently reported reductions in muscle perfusion rates and venous $\mathrm{pH}$ levels, and increased in arterial pressure, arterial-venous differences in oxygen concentration, and venous lactate levels, with little or no change in oxygen uptake rates (Holling and Verel, 1957; Astrand et al., 1968; Yamamoto and Fujita, 1983; Yata et al., 1985). When muscle blood flow is sufficiently impeded, sensations of fatigue and discomfort, and changes in other physiological metrics (e.g., electromyogram recordings) indicative of $\mathrm{LMF}$, are consistently encountered (Dorpat and Holmes, 1955; Holling and Verel, 1957; Start and Holmes, 1963; Mortimer et al., 1970; Yamamoto and Fujita, 1983). Posturing arms above heart level for sustained periods serves to reduce muscle perfusion, through increased hydrostatic and intramuscular pressures which, in turn, increase concentrations of noxious catabolites associated with signs and symptoms of LMF (Holling and Verel, 1957; Astrand et al., 1968; Yamamoto and Fujita, 1983). 
Holling and Verel (1957) reported that arm positions $50 \mathrm{~cm}$ above the heart reduced dynamic work capacity by 30 percent during a two-minute continuous exertion task. Reductions in arm work capacities with elevated arm postures occurred with workloads which could be maintained "indefinitely" when the arm was held at or below heart level. No reactive hyperemia was encountered upon cessation of arm elevations of durations up to 2 hours in length, and sensations of pain and discomfort were immediately relieved when the arm was brought down below the heart. Of significant interest to this study was the finding that symptoms continued undiminished in severity if the arm was passively supported in an elevated position. Thus, sustained elevation of the arm (i.e., increased hydrostatic pressures) during low-exertion manual activities may have great impact upon circulatory feed to musculature and subsequent symptomatology with or without taxing postural exertion capacity.

\section{Range-of-motion limits, passive strain, and discomfort}

Vertical or horizontal flexion or abduction of the arm towards its' range-of-motion (ROM) limits serves to increase tension in muscle and associated connective tissues. Basmajian (1961) suggested that discomfort experienced in some postures may be due simply to ligament and tendon strain, and not to ischemic muscle fatigue. He found complaints of discomfort could be obtained from muscle regions under stress, but which were electrically silent, and concluded that "muscles are spared where ligaments suffice".

Subsequent investigations point to the importance of connective tissue stress in provoking postural discomfort. Van Wely (1970), after examining several industrial work postures, concluded that excursions of limbs to near ROM limits, as well as increased load moments at the shoulder, were important determinants in worker complaints of discomfort in the upper extremity. Later, Chaffin (1973) showed experimentally that increasing shoulder load moments, through flexion, abduction, or arm extension, increased both onset and severity of subject reports of fatigue and discomfort. Cain (1973), investigated the nature of discomfort experienced during stressful exertions, also found sensations initially associated with ligament and tendon strain, which intensified following onset of muscle ischemia. Thus, upper extremity postures which passively stress involved tendons and ligaments may immediately provoke significant levels of discomfort even in the face of minimal exertion demands.

\section{Cross-modal gauging of fatigue and dis- comfort}

Intensity of fatigue or discomfort are positively correlated with exercise endurance time (Lloyd and McClaskey, 1971; Caldwell and Grossman, 1973; Kinsman et al., 1973; Kirk and Sadoyama, 1973), the degree of impedance of muscle blood flow (Caldwell and Smith, 1966), and with EMG and tremor indices of LMF (Lloyd et al., 1970). Matching intensities of fatigue or discomfort sensed with another sensory modality offers several advantages for assessment of posturally-based fatigue and discomfort. First, the method permits detection of discomfort due to ligamentous strain which metabolic indices of muscle fatigue (i.e., EMG, lactic acid, etc.) would not. Second, crossmodal techniques provide estimates of the magnitude of fatigue or discomfort experienced along an operationally relevant continuum. Third, the method appears to be useful in assessing postural stress even when postural exertions are not demanding (Kuorinka, 1981; Boussenna et al., 1982; Wiker, 1986). Fourth, cross-modal estimates of fatigue or discomfort have proven to be reliable for a wide range of muscle exertion levels or fatigue states (Caldwell and Smith, 1966; Weber et al., 1975). Finally, use of cross-modal matching techniques such as those described by Corlett and Bishop (1976) provide a means to simultaneously assess a large number of body regions and to determine which regions are most stressed.

\section{METHODS AND MATERIALS}

\section{Subjects}

Four subjects were selected from a group of volunteers in a graduate level engineering class. 
TABLE 1

Descriptive characteristics of subjects

\begin{tabular}{lrrrr}
\hline Metric & \multicolumn{2}{c}{ Subject } & & \\
\cline { 2 - 5 } & \multicolumn{1}{c}{ F1 } & \multicolumn{1}{c}{ F2 } & M1 & M2 \\
\hline Gender & F & F & M & M \\
Age (years) & 20 & 22 & 22 & 22 \\
Height (cm) & 160 & 159 & 186 & 168 \\
Weight (kg) & 59 & 60 & 74 & 67 \\
\hline
\end{tabular}

Subjects reported and appeared to be in good health, reported no remarkable history of musculoskeletal disease or injury, and participated on an informed consent and paid basis. We intentionally selected young adults for this study. Cumulative trauma and musculoskeletal pathos in the shoulder complex increase with age. These phenomena would serve to confound any relationship between postural fatigue or discomfort, and measured strength capability; thus, the probability of finding a relationship between strength capability and posturally-induced fatigue and discomfort would be greatest in a younger population. General descriptive anthropometric measurements are provided in Table 1.

\section{Apparatus and procedures}

Subjects performed a Fitts' reciprocal movement task using a pistol-gripped stylus held by their preferred hand. Each movement trial required subjects to continuously move the stylus back and forth between a pair of response plate holes, shown in Fig. 1, as rapidly and as accurately as possible for a period of $30 \mathrm{~s}$. Each movement trial was then followed by a $30 \mathrm{~s}$ rest period. Upon completion of a set of 12 trials (6 IDs $\times 2$ movement axes), subjects rested for 3

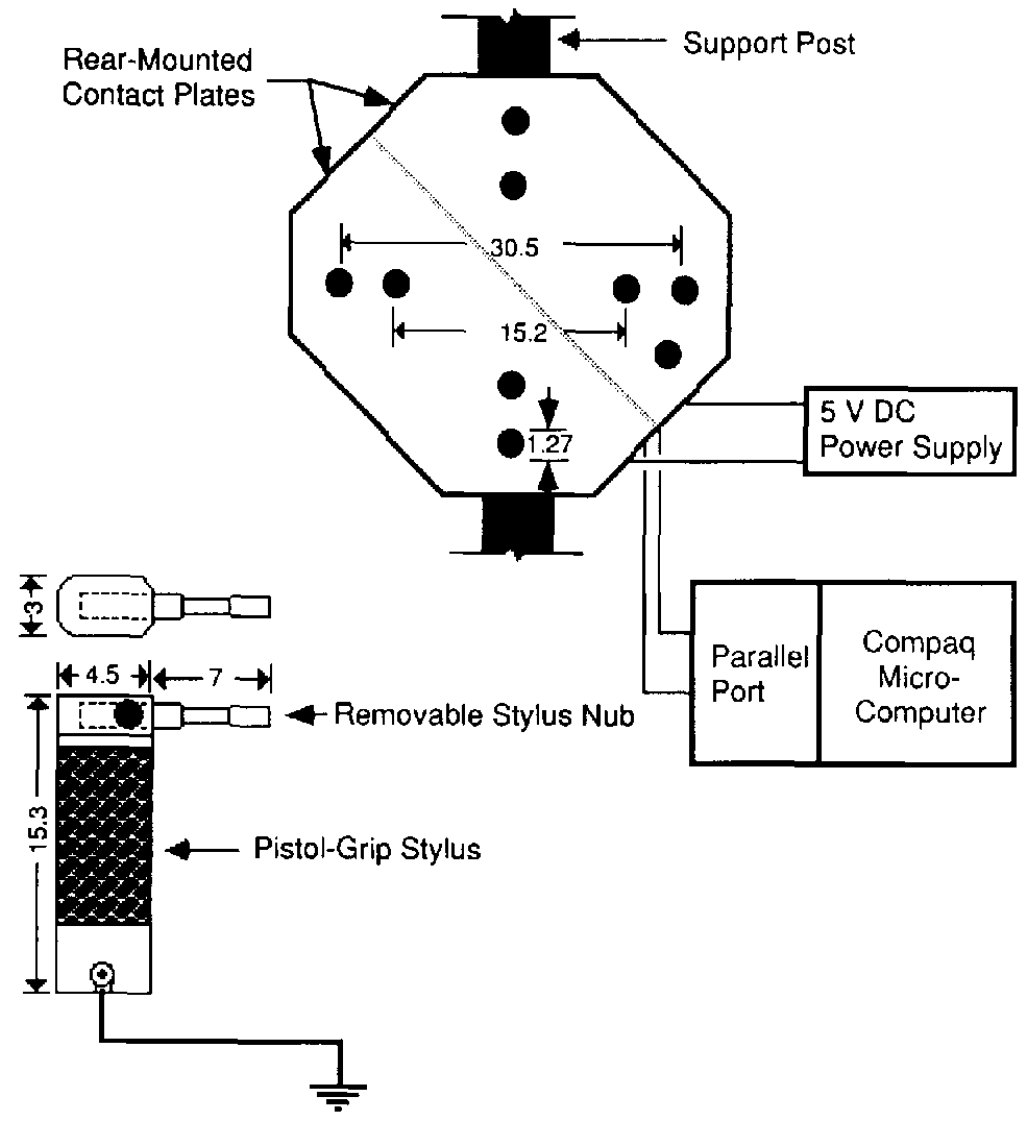

Fig. 1. Movement task plate, pistol-grip stylus, and data acquisition system used to record movement performance. Note that scalers are in centimeters and that the drawing is not to scale. 
minutes. Five sets of 12 trials were completed within a 75 minute period, on any given test day, with subjects assuming one of the 8 test posture and stylus weight combinations described in Fig. 2. Data collection for each subject was conducted over a three-week period and the order of treatments randomized. See Wiker et al. (1989) for a more detailed presentation of the movement task, movement analysis, and psychomotor performance findings.

The response plate was mounted upon a vertically sliding support beam, and was pivoted about the plate's horizontal axis to provide a working surface perpendicular to the longitudinal axis of the stylus when gripped by a seated subject of any particular anthropometry. Subjects sat upon an industrial stand/sit chair to control the distance between subject's shoulder and response plate, and to help stabilize the torso during arm movements.

The stylus consisted of a hollow pistol-shaped metal handle in which various stylus nubs could be exchanged. A racket-type wrapping tape covered the stylus handle to prevent slippage of the handle within the subject's hand. A removable lead core could be inserted into the center of the handle to provide additional weight as required. The stylus weighed $0.40 \mathrm{~kg}$. Adding the lead core to the stylus handle increased stylus weight to 1.50 $\mathrm{kg}$.

Figure 2 shows that the dominant arm was

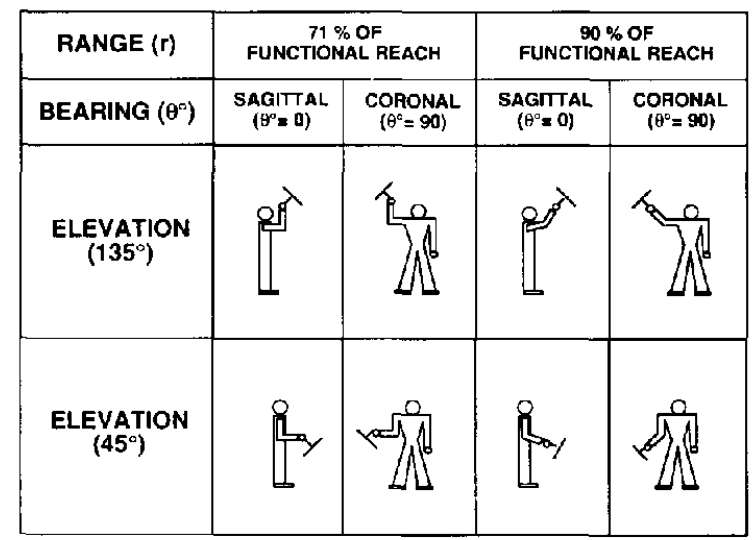

Fig. 2. Display of shoulder girdle postures and task variables investigated. postured either in the sagittal or coronal plane (i.e. bearing 0 or $90 \mathrm{deg}$, respectively), elevated 45 or $135 \mathrm{deg}$ from the side of the body in a flexed or abducted posture, and extension of the arm (i.e. range of grasp) was constrained to either 71 or 90 percent of the functional reach of the fully extended arm assuming a power grip (see Wiker (1986) for a detailed description and diagram of the functional reach measurement).

Reports of generalized, or global, and regional discomfort and fatigue were collected from subjects after completion of each set of 12 movement trials during the 3 minute rest period using a modified form of Corlett and Bishop's (1976) discomfort questionnaire. Corlett and Bishop's (1976) method, motivated by the work of Allen and Bennett (1958), requires subjects to successively rank-order body regions presented on a anthropomorphic diagram in terms of their discomfort, and to provide an overall assessment of discomfort using a seven-point scale using "extremely comfortable" and "extremely uncomfortable" as anchoring adjectives. Two sets of scores are obtained. The first score is the average global, or generalized, score of discomfort. The second score is the number of absolute discriminations of body regions perceived as differentially painful. For example, if subjects ranked body regions into four levels of discomfort (e.g., none or $0,1,2$, and 3) then the most painful regions would receive scores of 3 . The ranks for body parts are averaged between subjects, or are averaged across body regions within subjects.

Our modifications included segmenting the body into smaller functional regions, adding both right and left sides to the body, as shown in Fig. 3, and using a magnitude estimation score rather than ordinal ranking procedures. Subjects estimated the magnitude of fatigue and of discomfort for each symptomatic functional muscular region by indicating the length of a line anchored at the extremes by "no fatigue" and "extreme fatigue", or "no discomfort" and "extreme discomfort", respectively. The procedure was repeated to separately scale the magnitude of global fatigue and discomfort sensed.

Subjects were instructed to judge discomfort as sensations of sharp or dull pain, soreness, cramping, localized heat, throbbing, or aches as discomfort. Fatigue was judged as a sense of decline in 


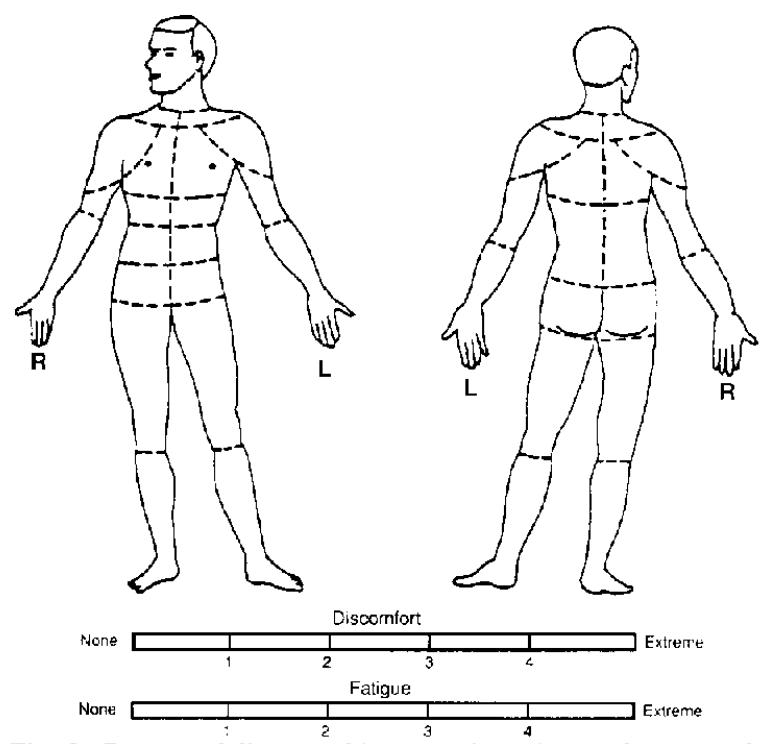

Fig. 3. Cross-modality matching questionnaire used to record global and regional sensations of fatigue and discomfort.

muscle contractility or strength, a feeling of weariness or exhaustion, or a reduction in one's endurance or stamina. Descriptions used were checked via interview for consistency with a subject's past experiences with fatigue and discomfort.

Each subject's isometric strength capability was measured for each direction of hand movement in each upper extremity posture studied save vertical extension and adduction movements wherein exertion magnitudes were confounded by the addition of body weight to force recordings. Strength tests were conducted following the procedures specified by Foulke and Keyserling (1979). Means of three replications served as strength estimates.

Experimentation commenced after several days of practice with the movement task and with fatigue and discomfort magnitude estimation procedures. The experimental protocol required that subjects be symptom-free for at least a twenty-four hours prior to any experiment session. Experimental apparatus and the stand/sit chair were adjusted to premarked positions based upon an individual's anthropometry and experimental posture required. Arm and shoulder posture, required for the particular experimental session, was initially confirmed using a set of calipers and goniometers, and then checked at 15 minute intervals as experiment sessions progressed.

\section{ANALYSIS AND RESULTS \\ Loci and magnitude of fatigue and dis- comfort experienced}

Reports of global fatigue and discomfort ranged between "none." and to near "extreme" during the course of this experiment. Reports of regional fatigue and discomfort were concentrated principally in the posterior-medial region of the deltoid, the upper trapezius, and to a much smaller degree, the anterior-medial region of the deltoid. Other functional anatomical regions were, for the most part, asymptomatic. Average reports of global, anterior medial deltoid, posterior medial deltoid, and upper trapezius reports of fatigue and discomfort are plotted across all experimental conditions in Figs. 4 and 5, respectively.

\section{Impact of postural and task variables upon postural fatigue and discomfort}

Magnitude estimates of fatigue and of discomfort, obtained during the second, third, and fourth rest periods *, were log-transformed to stabilize variances, and then analyzed using a repeatedmeasures mixed-effects analysis of variance (ANOVA) model in which changes in stylus weight were confounded with the three-way interaction between hand-height $\times$ hand-bearing $\times$ handrange to produce a half-fraction of a full-factorial experiment design. Subjects experienced all experimental conditions in random order and served as the random-effect blocking variable (see Montgomery (1976) for detailed presentations of the ANOVA model used).

The number and severity of reports of global fatigue were materially greater in magnitude when subjects performed movement tasks with hands elevated to $135 \mathrm{deg}$ (i.e., $45 \mathrm{deg}$ above the level of the shoulder $)(F(1,3)=10.2, \mathrm{MSE}=3.7, p<0.05)$. The sample size was insufficient to interpret the statistical significance of the effects of stylus weight $(F(1,3)=3.0, \quad \mathrm{MSE}=1.18, \quad p=0.18)$, or

\footnotetext{
* Reports obtained following the first and last set of trials were excluded a priori from analyses to minimize any potential warm-up and end effects.
} 


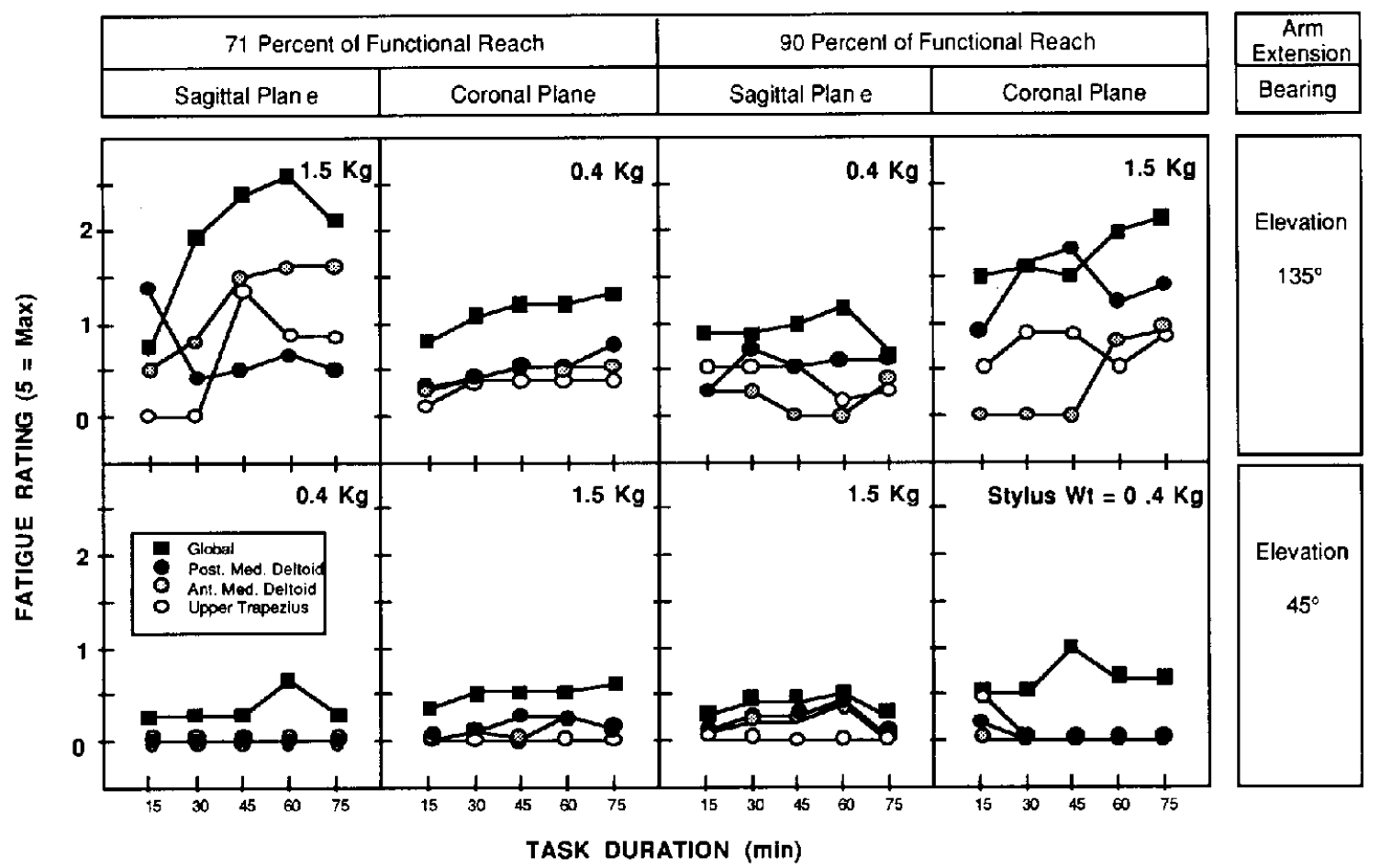

Fig. 4. Average magnitudes of global fatigue plotted against hand elevation, bearing, arm extension, and task duration.

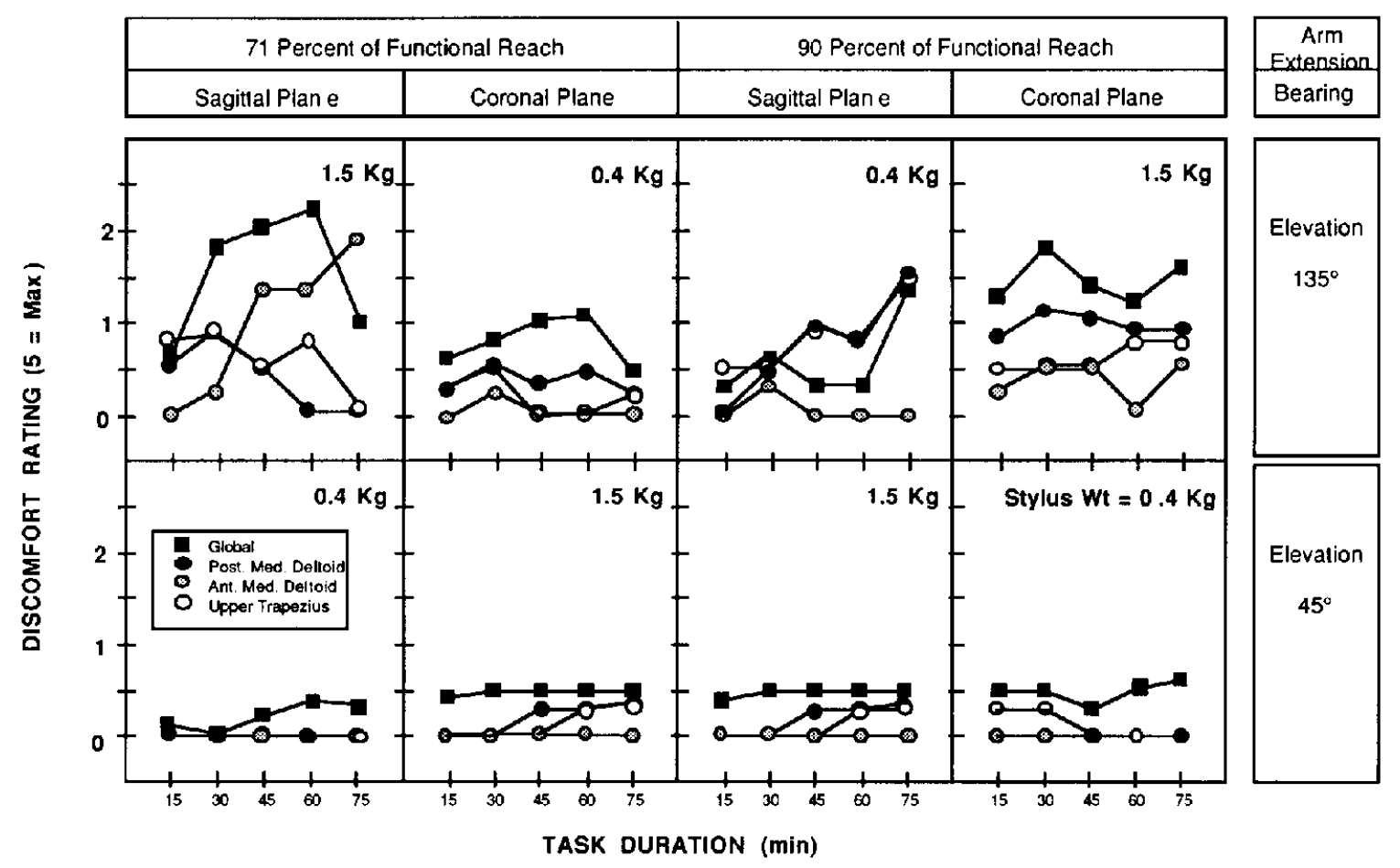

Fig. 5. Average global reports of discomfort plotted against hand elevation, bearing, arm extension, and task duration. 
the interaction between hand elevation and stylus weight $(F(1,3)=6.9, \mathrm{MSE}=1.2, p=0.08)$ upon reports of fatigue (i.e., statistical power was less than 0.90 for an increase of 10 percent of the magnitude range). However, changes in hand bearing, arm extension, task duration, and all remaining interactions showed no impact upon global sensations of fatigue ( $p>0.05$, power $>$ 0.90 ). See Wiker (1986) for a complete presentation of ANOVA tables for all tests reported in this paper.

Reports of global discomfort were also more severe when subjects performed the movement task in the elevated arm posture $(F(1,3)=9.6$, MSE $=1.65, p<0.05)$. The sample size was again insufficient to interpret the statistical significance of increased stylus weight $(F(1,3)=4.5, \mathrm{MSE}=$ $1.71, p=0.12$ ), as well as the interaction between hand elevation and stylus weight $(F(1,3)=4.6$, $\mathrm{MSE}=1.33, p=0.16$ ), upon reports of discomfort. Changes in hand bearing, arm extension, task duration, as well as the interactions studied, showed no effect upon sensations of global discomfort $(p>0.05$, power $>0.90)$.

Regional reports of fatigue and discomfort were typically smaller in magnitude than global reports. In many treatment combinations, particularly with low hand elevations, or with initial samples in test sessions, fatigue or discomfort were not reported. The number of treatment combinations in which regional discomfort and fatigue were not experienced was sufficiently large that a general linear test was used for hypotheses testing; a regression approach to an ANOVA in which only non-zero responses were included in the analysis. Tests were conducted on main and two-way interaction ef- fects following the procedures of Neter and Wasserman (1974).

Greater magnitudes of fatigue in the trapezius muscle region were found with arm elevation $(F(1,23)=17.7, \mathrm{MSE}=0.64, p<0.05)$, with arm elevation when manipulating the heavier stylus $(F(2,22)=0.59 .5, \mathrm{MSE}=0.18, p<0.05)$, and with progression of the task when arms were elevated $(F(9,15)=3.6, \mathrm{MSE}=0.54, p<0.05)$. No significant changes in reports of fatigue in the upper trapezius were found with changes in hand bearing, arm extension, or task duration $(p>0.10)$.

Reports of fatigue in the anterior-medial and posterior-medial deltoid regions were greater in magnitude when stylus weight was increased $(F(1,25)=6.8, \mathrm{MSE}=0.53, p<0.05$ and $F(1,49)$ $=6.6, \mathrm{MSE}=0.82, p<0.05$, respectively); with heavier styli producing much greater fatigue in elevated arm postures $(F(2,24)=4.6, \mathrm{MSE}=0.50$, $p<0.05$ and $F(2.47)=3.8, \mathrm{MSE}=0.78, p<0.05$, respectively). No effects were found with changes in hand bearing, arm extension, or task duration $(p<0.10)$.

It was not possible to perform a meaningful statistical analysis on reports of discomfort in the anterior medial deltoid region. Only 13 out of 160 questionnaires contained nonzero reports of discomfort in the anterior medial deltoid region. All nonzero reports were obtained in elevated arm postures, and 11 out of 13 reports occurred when subjects were performing movements with the 1.5 $\mathrm{kg}$ stylus.

Upper trapezius discomfort increased with arm elevation $(F(1,28)=5.1, \mathrm{MSE}=0.40, p<0.05)$. No other main effects, or two-way interactions, were found to be significant $(p>0.10)$.

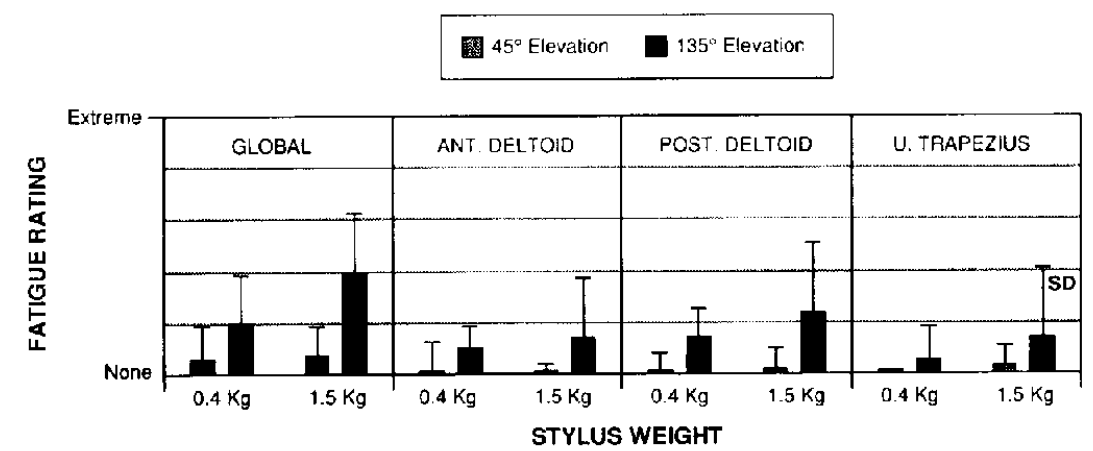

Fig. 6. Average magnitude of global and regional reports of fatigue plotted against hand elevation and stylus weight. 


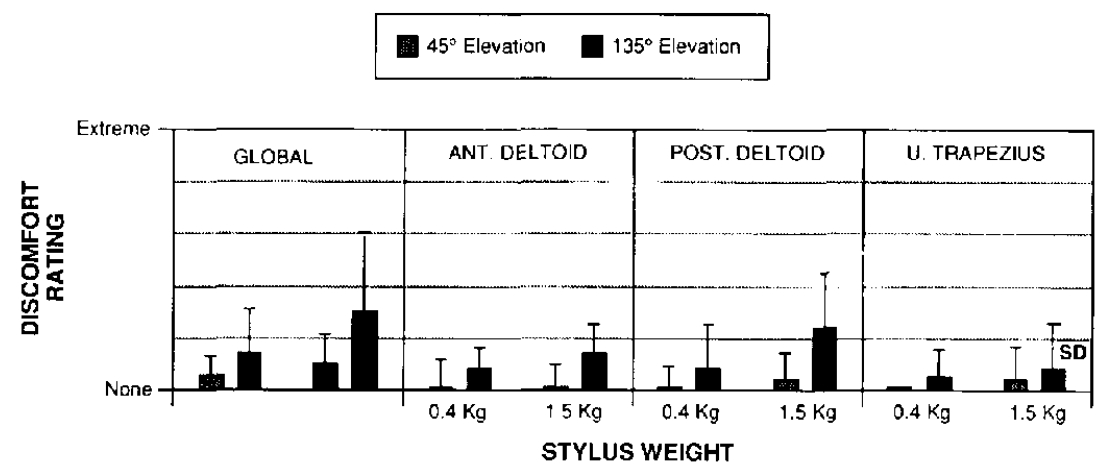

Fig. 7. Average magnitude of global and regional reports of discomfort plotted against hand elevation and stylus weight.

Posterior-medial deltoid region discomfort increased with added stylus weight $(F(1,30)=5.1$, $\mathrm{MSE}=0.35, p<0.05)$; particularly with elevated arm postures $(F(2,29)=4.2, \quad \mathrm{MSE}=0.35, \quad p<$ $0.05)$. No other main effects, or two-way interactions, were found to be significant $(p>0.10)$.

In Figs. 6 and 7 average magnitudes of reports of global, upper trapezius, anterior-medial deltoid, and posterior-medial deltoid regional fatigue and discomfort are respectively collapsed across hand elevation and stylus weight conditions examined.

\section{Relationships among reports of fatigue and discomfort}

Correlations were computed between mean reports of global and regional fatigue and discomfort obtained for each subject in each test posture and stylus weight combination. Results, presented in Table 2, show correlations between reports of global fatigue and global discomfort, and between estimates of fatigue and discomfort within a functional muscle grouping, were typically strong. Increasing reports of global discomfort or fatigue were associated with greater numbers of symptomatic functional muscle regions which is borne out by the number and magnitude of statistically significant intercorrelations obtained.

\section{Shoulder strength, moments, and pos- tural fatigue and discomfort}

Measurements of subject horizontal flexion and extension, and vertical flexion isometric strength capability, within each test posture, are summarized in Table 3. External moments at the glenohumeral articulation were estimated using the subject's measured anthropometry, link angles recorded while assuming postures examined in this experiment, predictions of link mass and centers of mass from Dempster (1955), and procedures described by Chaffin and Andersson (1984). Predicted external shoulder moments are summarized across subjects and test postures in Table 4.

Table 5 presents correlations computed between mean reports of fatigue and discomfort obtained from each test posture and stylus weight combination, postural and task variables which

TABLE 2

Correlations among reports of discomfort and fatigue

\begin{tabular}{|c|c|c|c|c|c|c|c|c|}
\hline & Variable & 1 & 2 & 3 & 4 & 5 & 6 & 7 \\
\hline 1 & Global Discomfort (D.) & - & - & - & - & - & - & - \\
\hline 2 & Global Fatigue (F.) & 0.76 & - & - & - & - & - & - \\
\hline 3 & Ant. Med. Deltoid D. & 0.36 & 0.48 & - & - & - & - & - \\
\hline 4 & Upper Trapezius D. & 0.37 & 0.38 & 0.16 & - & - & - & - \\
\hline 5 & Post. Med. Deltoid D. & 0.63 & 0.66 & 0.43 & 0.32 & - & - & - \\
\hline 6 & Ant. Med. Deltoid F. & 0.39 & 0.49 & 0.82 & 0.33 & 0.30 & - & - \\
\hline 7 & Upper Trapezius F. & 0.62 & 0.60 & 0.21 & 0.49 & 0.56 & 0.14 & - \\
\hline 8 & Post. Med. Deltoid F. & 0.70 & 0.67 & 0.04 & 0.38 & 0.70 & 0.11 & 0.77 \\
\hline
\end{tabular}

Note: Boldface correlations $(N=32, p<0.05)$. 
TABLE 3

Average isometric strength capability of subjects

\begin{tabular}{|c|c|c|c|c|c|c|c|}
\hline \multirow[t]{2}{*}{ Subject } & \multicolumn{3}{|c|}{ Arm posture } & \multicolumn{3}{|c|}{ Strength (N) } & \multirow{2}{*}{$\begin{array}{l}\text { Stylus wt. } \\
\% \text { MVC fo } \\
\text { Vertical } \\
\text { flexion }\end{array}$} \\
\hline & $\begin{array}{l}\text { Hand } \\
\text { elevation a }\end{array}$ & $\begin{array}{l}\text { Hand } \\
\text { bearing }\end{array}$ & $\begin{array}{l}\text { Hand } \\
\text { range }(\%)\end{array}$ & $\begin{array}{l}\text { Horz. } \\
\text { flexion }\end{array}$ & $\begin{array}{l}\text { Horz. } \\
\text { extension }\end{array}$ & $\begin{array}{l}\text { Vertical } \\
\text { flexion }\end{array}$ & \\
\hline \multirow[t]{6}{*}{ F1 } & 45 & 0 & 71 & 72 & 66 & 143 & 3 \\
\hline & 45 & 0 & 90 & 55 & 63 & 102 & 15 \\
\hline & 45 & 90 & 71 & 70 & 55 & 134 & 11 \\
\hline & 45 & 90 & 90 & 60 & 54 & 134 & 3 \\
\hline & & & Mean & 64.3 & 59.5 & 128.3 & 7.9 \\
\hline & & & St. Dev. & 8.1 & 5.9 & 18.0 & 6.0 \\
\hline \multirow[t]{6}{*}{$F 1$} & 135 & 0 & 71 & 36 & 49 & 108 & 14 \\
\hline & 135 & 0 & 90 & 36 & 60 & 67 & 6 \\
\hline & 135 & 90 & 71 & 84 & 54 & 104 & 4 \\
\hline & 135 & 90 & 90 & 55 & 51 & 93 & 16 \\
\hline & & & Mean & 52.8 & 53.5 & 93.0 & 9.9 \\
\hline & & & St. Dev. & 22.7 & 4.8 & 18.5 & 6.0 \\
\hline \multirow[t]{6}{*}{ M1 } & 45 & 0 & 71 & 160 & 111 & 182 & 2 \\
\hline & 45 & 0 & 90 & 118 & 102 & 170 & 9 \\
\hline & 45 & 90 & 71 & 111 & 81 & 130 & 12 \\
\hline & 45 & 90 & 90 & 91 & 106 & 143 & 3 \\
\hline & & & Mean & 120.0 & 100.0 & 156.3 & 6.3 \\
\hline & & & St. Dev. & 29.0 & 13.2 & 23.9 & 4.6 \\
\hline \multirow[t]{6}{*}{ M1 } & 135 & 0 & 71 & 129 & 84 & 134 & 11 \\
\hline & 135 & 0 & 90 & 118 & 67 & 116 & 3 \\
\hline & 1.35 & 90 & 71 & 127 & 90 & 99 & 4 \\
\hline & 135 & 90 & 90 & 106 & 100 & 164 & 9 \\
\hline & & & Mean & 120.0 & 85.3 & 128.3 & 6.8 \\
\hline & & & St. Dev. & 10.5 & 13.8 & 27.8 & 3.8 \\
\hline \multirow[t]{6}{*}{$\mathrm{F} 2$} & 45 & 0 & 71 & 64 & 64 & 157 & 3 \\
\hline & 45 & 0 & 90 & 60 & 60 & 149 & 10 \\
\hline & 45 & 90 & 71 & 90 & 72 & 99 & 15 \\
\hline & 45 & 90 & 90 & 57 & 57 & 90 & 4 \\
\hline & & & Mean & 67.8 & 63.3 & 123.8 & 8.0 \\
\hline & & & St. Dev. & 15.1 & 6.5 & 34.1 & 5.7 \\
\hline \multirow[t]{6}{*}{$\mathrm{F} 2$} & 135 & 0 & 71 & 63 & 49 & 133 & 11 \\
\hline & 135 & 0 & 90 & 46 & 42 & 93 & 4 \\
\hline & 135 & 90 & 71 & 79 & 75 & 116 & 3 \\
\hline & 135 & 90 & 90 & 70 & 63 & 91 & 16 \\
\hline & & & Mean & 64.5 & 57.3 & 108.3 & 8.9 \\
\hline & & & St. Dev. & 14.0 & 14.7 & 20.0 & 6.2 \\
\hline \multirow[t]{6}{*}{ M2 } & 45 & 0 & 71 & 127 & 81 & 154 & 3 \\
\hline & 45 & 0 & 90 & 93 & 78 & 121 & 12 \\
\hline & 45 & 90 & 71 & 93 & 75 & 122 & 12 \\
\hline & 45 & 90 & 90 & 82 & 67 & 109 & 4 \\
\hline & & & Mean & 98.8 & 75.3 & 126.5 & 7.7 \\
\hline & & & St. Dev. & 19.5 & 6.0 & 19.3 & 5.3 \\
\hline \multirow[t]{6}{*}{$\mathrm{M} 2$} & 135 & 0 & 71 & 82 & 57 & 67 & 22 \\
\hline & 135 & 0 & 90 & 86 & 79 & 78 & 5 \\
\hline & 135 & 90 & 71 & 91 & 82 & 121 & 3 \\
\hline & 135 & 90 & 90 & 82 & 78 & 97 & 15 \\
\hline & & & Mean & 85.3 & 74.0 & 90.8 & 11.5 \\
\hline & & & St. Dev. & 4.3 & 11.5 & 23.7 & 9.0 \\
\hline
\end{tabular}

0 = Sagittal plane

b $90=$ Coronal plane. 
TABLE 4

Estimated external moments at subjects' shoulder and elbow joints for given arm postures and stylus weights

\begin{tabular}{|c|c|c|c|c|c|c|c|c|}
\hline \multirow{3}{*}{$\begin{array}{l}\text { Hand elevation }^{\circ} \\
\text { Arm extension }(\%) \\
\text { Stylus weight }(\mathrm{kg})\end{array}$} & \multicolumn{4}{|l|}{45.0} & \multicolumn{4}{|l|}{135.0} \\
\hline & 71.0 & 71.0 & 90.0 & 90.0 & 71.0 & 71.0 & 90.0 & 90.0 \\
\hline & 0.4 & 1.5 & 0.4 & 1.5 & 0.4 & 1.5 & 0.4 & 1.5 \\
\hline \multicolumn{9}{|l|}{ Subject FI } \\
\hline Arcromion-Elbow (cm) & 29.0 & - & - & - & - & - & - & - \\
\hline Elbow-Grip (cm) & 33.0 & - & - & - & - & - & - & - \\
\hline$d^{\circ} \mathrm{a}$ & -96.0 & -96.0 & -74.0 & -74.0 & -6.0 & -6.0 & 16.0 & 16.0 \\
\hline$\beta^{\circ b}$ & -2.0 & -2.0 & -20.0 & -20.0 & 88.0 & 88.0 & 70.0 & 70.0 \\
\hline Shoulder moment $(\mathrm{Nm})$ & -2.9 & -5.8 & -5.7 & -9.6 & -8.1 & -11.1 & -9.0 & -12.8 \\
\hline Elbow moment $(\mathrm{Nm})$ & -3.8 & -7.0 & -3.5 & -6.6 & -0.1 & -0.2 & -1.3 & -2.4 \\
\hline \multicolumn{9}{|l|}{ Subject F2 } \\
\hline Arcromion-Elbow $(\mathrm{cm})$ & 28.0 & - & - & - & - & - & - & - \\
\hline Elbow-Grip & 28.0 & - & - & - & - & - & - & - \\
\hline$\partial^{\circ}$ & -93.0 & -93.0 & -73.0 & -73.0 & -3.0 & -3.0 & 17.0 & 17.0 \\
\hline$\beta^{\circ}$ & -3.0 & -3.0 & 17.0 & 17.0 & 93.0 & 93.0 & 73.0 & 73.0 \\
\hline Shoulder moment $(\mathrm{Nm})$ & -2.8 & -5.5 & -5.5 & -9.0 & -7.9 & -10.5 & -8.7 & -12.2 \\
\hline Flbow moment $(\mathrm{Nm})$ & -3.3 & -6.1 & -5.1 & -5.8 & -0.2 & -0.3 & -1.0 & -1.8 \\
\hline \multicolumn{9}{|l|}{ Subject $M I$} \\
\hline Arcromion-Elbow (cm) & 34.0 & - & - & - & - & - & - & - \\
\hline Elbow-Grip (cm) & 38.0 & - & - & - & - & - & - & - \\
\hline$\partial^{\circ}$ & -95.0 & -95.0 & -74.0 & -74.0 & -5.0 & -5.0 & 16.0 & 16.0 \\
\hline$\beta^{\circ}$ & 2.0 & 2.0 & 20.0 & 20.0 & 88.0 & 88.0 & 70.0 & 7.0 \\
\hline Shoulder moment (Nm) & -4.0 & -7.5 & -8.0 & -12.6 & -11.7 & -15.2 & -12.9 & -17.4 \\
\hline Elbow moment $(\mathrm{Nm})$ & -5.0 & -8.8 & -4.8 & -8.3 & -0.2 & -0.3 & -1.7 & -3.0 \\
\hline \multicolumn{9}{|l|}{ Subject $M 2$} \\
\hline Arcromion-Elbow $(\mathrm{cm})$ & 33.0 & - & - & - & - & - & - & - \\
\hline Elbow-Grip (cm) & 33.0 & - & - & - & - & - & -1 & - \\
\hline$\partial^{\circ}$ & -91.0 & -91.0 & -71.0 & -71.0 & -1.0 & -1.0 & 18.0 & 18.0 \\
\hline$\beta^{\circ}$ & -1.0 & -1.0 & 18.0 & 18.0 & 91.0 & 91.0 & 72.0 & 72.0 \\
\hline Shoulder moment (Nm) & -3.9 & -7.1 & -7.2 & -11.4 & -10.3 & -13.5 & -11.2 & -15.3 \\
\hline Elbow moment (Nm) & -4.1 & -7.4 & -3.9 & -7.0 & -0.1 & -0.2 & -1.3 & -2.3 \\
\hline Mean elbow moment $(\mathrm{Nm})$ & -4.1 & -7.3 & -4.3 & -6.9 & -0.2 & -0.3 & -1.3 & -2.4 \\
\hline S.D. & 0.7 & 1.1 & 0.8 & 1.0 & 0.1 & 0.1 & 0.3 & 0.5 \\
\hline Mean shoulder moment $(\mathrm{Nm})$ & -3.4 & -6.5 & -6.6 & -10.7 & -9.5 & -12.6 & -10.5 & -14.4 \\
\hline S.D. & 0.6 & 1.0 & 1.2 & 1.7 & 1.8 & 2.2 & 2.0 & 0.4 \\
\hline
\end{tabular}

$\partial^{\circ}=$ angle of the upper arm above $(+)$ or below $(-)$ the horizontal axis.

${ }^{\mathrm{b}} \beta^{\circ}=$ angle of the forearm above (+) or blow (-) the horizontal axis.

affected shoulder moments and strength, predicted shoulder external moments, and shoulder flexion or abduction isometric strength capability. Though elevation of the arm through flexion in the sagittal plane, or abduction in the coronal plane, was moderately associated with decrements in shoulder flexion or abduction strength capability, or postural exertion capacity, no statistically or materially meaningful correlations were found between global or regional reports of fatigue or discomfort.
Hand elevation was significantly correlated with shoulder external moments, and both showed meaningful and direct relationships between all global and regional reports of fatigue and of discomfort. Shoulder external moments exhibited consistently stronger relationships than did hand elevation. Larger shoulder external moments were encountered with arm extension or added stylus weight; however, the consequences were small in comparison with those of hand elevation, and no 
TABLE 5

Correlations among hand elevation, arm extension, stylus weight, shoulder isometric flexion strength, estimated external shoulder moments, and reports of discomfort and fatigue

\begin{tabular}{rllcrrrr}
\hline & Variable & 1 & 2 & 3 & 4 & 11 & 12 \\
\hline 1 & Hand Elevation & - & - & - & - & $-\mathbf{0 . 4 9}$ & 0.16 \\
2 & Arm Fxtension & - & - & - & -0.20 & 0.07 \\
3 & Stylus Weight & - & - & - & - & - & - \\
4 & Shoulder Moment & $\mathbf{0 . 6 6}$ & $\mathbf{0 . 3 8}$ & $\mathbf{0 . 4 9}$ & - & -0.26 & 0.16 \\
5 & Global Fatigue (F.) & $\mathbf{0 . 5 9}$ & 0.05 & 0.28 & $\mathbf{0 . 7 0}$ & 0.02 & 0.29 \\
6 & Upper Trapezius F. & $\mathbf{0 . 3 6}$ & -0.10 & 0.19 & $\mathbf{0 . 5 6}$ & 0.10 & 0.19 \\
7 & Post. Med. Deltoid F. & $\mathbf{0 . 4 9}$ & 0.07 & 0.24 & $\mathbf{0 . 7 2}$ & 0.17 & 0.13 \\
8 & Global Discomfort (D.) & $\mathbf{0 . 4 1}$ & 0.08 & 0.26 & $\mathbf{0 . 6 5}$ & 0.16 & $\mathbf{0 . 2 1}$ \\
9 & Upper Trapezius D. & $\mathbf{0 . 3 9}$ & 0.19 & 0.01 & $\mathbf{0 . 5 2}$ & -0.06 & $\mathbf{0 . 1 6}$ \\
10 & Post. Med. Deltoid D. & $\mathbf{0 . 4 2}$ & 0.16 & 0.24 & $\mathbf{0 . 6 5}$ & 0.10 & 0.25 \\
11 & Shoulder Isometric Strength & & & & & & \\
12 & Stylus Wt: Shoulder Flexion Strength Ratio & & & & &
\end{tabular}

Note: Boldface correlations $(N=32, p<0.05)$.

relationships were found between either variable and reports of global or regional fatigue and discomfort.

\section{DISCUSSION}

Differences in upper extremity strength capability found among test subjects, or among different arm postures within a subject, were unrelated to differences in the severity of reports of postural fatigue or of discomfort when exertions were small (i.e., less than 15\% MVC). Moreover, reports of fatigue and discomfort were not materially affected by changes in arm posture which did not significantly increase arm elevation. If arms were postured above shoulder-level, substantial complaints were reported in less than one-hour in spite of low-level exertions (i.e., mean exertions of 9.1 percent of MVC) and comparatively generous rest intervals. Elevation of the dominant hand to 135 deg (i.e., 45 deg above shoulder-level) and manipulation of a $1.5 \mathrm{~kg}$ stylus, produced averaged reports of global fatigue and of discomfort which approached 40 percent of the available response range; ranging between no symptoms and extreme sensations. No significant accumulation of fatigue was observed during the 75 minute test period; a result which may be attributed to generous rest periods (e.g., 15 minute performance cycles contained 9 minutes of rest).
Strength capacity, as shown in this and many other studies, is significantly influenced by changes in arm posture. However, elevation of the arm, regardless of other posture variables (e.g., alignment within the sagittal or coronal plane), results in significant and comparable levels of co-contraction of, and increased mechanical strain and intramuscular pressure within, musculature and connective tissues comprising the shoulder complex (Codman, 1934; Inman et al., 1944; Dempster, 1965). In addition to mechanical strain, and the vascular challenges produced by added intramuscular pressure, raising the arm above heart-level serves to progressively increase hydrostatic impedance to circulatory feed of shoulder musculature; again, regardless of the arm's relative alignment about the body (Barcroft and Millan, 1939; Holling and Verel, 1957; Start and Holmes, 1963; Astrand et al., 1968; Kadefors et al., 1968; Jarvholm et al., 1988; Yata et al., 1985). Thus, while postural changes in the elevated arm produced material differences in strength capability, levels of mechanical and vascular stress in muscle or connective tissue, leading to symptoms of fatigue and discomfort, may not have changed significantly with changes in arm bearing or extension. Thus, unless exertion demands were high, relationships between exertion level and fatigue symptomatology would be undermined both within and between individuals and experimental findings, such as ours, would be encountered. 
The remainder of our findings were mostly supportive of past studies of postural discomfort. Postures which were fatiguing were also uncomfortable, and severity of global reports increased with the number of regions reported as fatigued or uncomfortable. This result is in agreement with Corlett and Bishop's (1976) study of postural discomfort, with findings of a recent perceived exertion experiment (Robertson et al., 1979), and with several sensory physiology experiments in which perceptions of stimulus severity increased when areas of stimulation were increased (Mountcastle, 1974). However, contrary to Corlett and Bishop (1976), we found that global reports of discomfort or of fatigue were strongly related to the severity of symptoms experienced in specific muscle groups; namely, the upper trapezius and deltoids. Differences in the assessment methods used may account for the apparent contradiction in findings. We asked subjects to match the severity of fatigue or discomfort for each symptomatic region, as well as globally, using a magnitude estimation technique. Corlett and Bishop, on the other hand, asked subjects to segregate regions of discomfort into groups of equivalent severity, and that differences in severity between groupings should be distinct. The range of regional severity was, therefore, dependent upon the number of distinct groups obtained. The rank-order scaling approach, limited range in ranks, along with the use of much larger body regions in their assessments, may account for weaker relationships found in Corlett and Bishop's study.

From a job design perspective, results of this experiment do not support a strategy of relying solely upon upper extremity strength capability to choose from among candidate extremity postures which include over-shoulder work, or the contention that stronger workers are better suited to perform light-weight manual assembly tasks with sustained elevated arm postures. Moreover, our results show that postures which may appear awkward (e.g., working with hands postured within the coronal plane near waist-level) did not necessarily provoke significant fatigue and discomfort. Though the probability that we failed to detect such relationships is low (Type II error $<0.10$ ), the reader is reminded that our subject population was small in number and was selected for their youth and absence of cumulative damage to the shoulder complex. Older workers, or workers who have experienced longterm postural or exertion stress or pathology in the shoulder, would probably demonstrate less tolerance for the conditions tested in this study. Nevertheless, our findings should encourage ergonomists to eliminate overhead work wherever possible, even in light-weight manual assembly environments, and to extend their analyses beyond anthropometrics and upper extremity strength capability when evaluating or specifying arm and shoulder postures in lightweight manual assembly environments.

\section{CONCLUSIONS AND RECOMMENDA- TIONS}

Differences in exertion capacity found between subjects, or among postures within a subject, showed no relationship with either the locus or magnitude of global or regional fatigue and discomfort experienced in this study. Periodic sustained elevation of the dominant hand above shoulder level provoked substantial postural discomfort and fatigue in light-weight manual assembly environs in spite of low-level exertion demands (i.e., less than 10 percent MVC). This finding supports Bjorksten and Jonsson's (1977) recommendation that static or postural exertions should be much less than the 15 percent of MVC which is found in most extant engineering design guides.

Our findings suggest that evaluation or specification of light-weight repetitive working postures requires careful consideration of a number of factors which go well beyond the determination of an individual's functional reach and strength capacity. It is not unusual to find stronger workers selected for manual assembly overhead or using other awkward upper extremity postures. Neither characterization of each test subject's isometric strength capability, which exceeded population norms summarized by Chaffin and Andersson (1984), and which differed considerably between subjects as well as among postures within subjects, or computation of external shoulder load moments, were of value in predicting or gauging onset of severity of fatigue and discomfort experienced in elevated arm postures. Though the probability that we failed to detect such relation- 
ships is low (Type II error $<0.10$ ), independent verification of our findings using a broader sample of the eligible working population is warranted. Until then, our findings should encourage ergonomists to eliminate overhead work even in light-weight manual assembly environments, and to extend their analyses beyond evaluation of anthropometrics and upper extremity strength capability when specifying arm and shoulder postures in light-weight manual assembly environments.

\section{REFERENCES}

Allen, P.S. and Bennett. E., 1958. Forced choice ranking as a method of evaluating psycho-physiological feelings. USAF WADC Tech. Report No. 58.

Astrand, 1.. Guharay, A. and Wahren, J., 1968. Circulatory response to arm exercise with different arm positions. Acta Med. Scand., 2: 528-533.

Basmajian, J.V., 1961. Weight-bearing by ligaments and muscles. Can. J. Surg., 4: 166-170.

Barcroft, H. and Millan, J.L.E., 1939. The blood flow through muscle during sustained contraction. J. Physiol. (London), 97: 17-31.

Bjorksten, M. and Jonsson, B., 1977. Fndurance limit of force in long-term intermittent static contractions. Scand. J. Work Environ. Health, 3: 23-27.

Boussenna, M., Corlett, F.N. and Pheasant, S.T., 1982. The relation between discomfort and postural loading at the joints. Ergonomics, 25(4): 315-322.

Cain, W.S., 1973. Nature of perceived effort and fatigue: Roles of strength and blood flow in muscle contractions. J. Mot. Behav., 5(1): 33-47.

Caldwell, L.S. and Smith, R.P., 1966. Pain and endurance of isometric muscle contractions. J. Eng. Psychol., 5(1): 25-32.

Caldwell, L.S. and Grossman, E.E., 1973. Effort scaling of isometric muscle contractions. J. Mot. Behav., 5(1): 9-16.

Chaffin, D.B., 1973. Localized muscle fatigue-definition and measurement. J. Occup. Med., 15(4): 346-354.

Chaffin, D.B. and Andersson, G.. 1984. Occupational Biomechanics. Wiley, New York

Codman, E.A., 1934. The Shoulder. Thomas Todd, Boston.

Corlett, E.N. and Bishop, R.P., 1976. A technique for assessing postural discomfort. Ergonomics, 19(2): 175-182.

Dempster, W.T., 1955. Space requirements of the seated operator. Technical Report WADC-TR-55-159, Wright Patterson Air Force Base, Dayton, OH.

Dempster, W.T., 1965. Mechanisms of shoulder movement. Arch. Phys. Med. Rehabil., 46(1-A)L 49-69.

Dorpat, T.L. and Holmes, T.H., 1955. Mechanisms of skeletal muscle pain and fatigue. AMA Arch. Neurol. Psychiat., 74: $628-640$.

Foulke, J.A. and Keyserling. W.M., 1979. Force monitor: A system for human strength measurement. Center for Ergonomics Technical Report, Ann Arbor, MI.

Ilolling, H.F. and Verel, D., 1957. Circulation in the clevated forearm. Clin. Sci., 16: 197-213.

Inman. V.T.. Saunders, J.B. and Abbott, L.C., 1944. Observa- tions on the function of the shoulder joint. J. Bone Joint Surg., 26(1): 1-30.

Jarvholm, U., Herberts, P., Kadefors, R., Korner, L., Palmerud, G. and Styf, J., 1988. Intramuscular pressure in the supraspinatus muscle. J. Orthop. Res., 6(2): 230-238.

Kadefors, R., Kaiser, E. and Petersen, I., 1968. Dynamic spectrum analysis of myopotentials with special reference to muscle fatigue. Electromyography, 8: 39-74.

Kinsman, R.A., Weiser, P.C. and Stamper, D.A., 1973. Multidimensional analysis of subjective symptomatology during prolonged strenuous exercise. Ergonomics, 16(2): 211-226.

Kirk, N.S. and Sadoyama. T., 1973. A relationship between endurance and discomfort in static work. Ergonomics, 19: 2.

Kuorinka, I., 1981. Discomfort and motor skills in semi-paced tasks. In: G. Salvendy and M.J. Smith (Eds.), Machine Pacing and Occupational Stress. Taylor \& Francis, London, pp. 143-150.

Lloyd, A.J., Voor, J.H. and Thieman, T.J., 1970. Subjective and electromyographic assessment of isometric muscle contractions. Ergonomics, 13(6): 685-691.

Lloyd, A.J. and McClaskey, E.B., 1971. Subjective assessment of effort in dynamic work. J. Mot. Behav. 3(1): 49-56.

Montgomery, D.C., 1976. Design and Analysis of Experiments. Wiley, New York.

Mortimer, J.T., Magnusson, R. and Petersen, I., 1970. Conduction velocity in ischemic muscle: Effect on EMG frequency spectrum. Amer. J. Physiol., 219(5): 1324-1329.

Mountcastle, V.B., 1974. Pain and temperature sensibilities. In: V.B. Mountcastle (Ed.), Medical Physiology. C.V. Mosby Co., Saint Louis, 13th edn., pp. 348-383.

Neter, J. and Wasserman, W., 1974. Applied Linear Statistical Models. Irwin, Homewood, IL.

Robertson, R.J., Gillespie, R.L., McCarthy, J. and Rose, K., 1979. Differentiated perceptions of exertion: Part I. Mode of integration of regional signals. Percept. Mot. Skills, 49; 683-689.

Rohmert, W., 1973. Problems in determining rest allowances. Appl. Ergon., 4(2): 91-95.

Start, K.B. and Holmes, R., 1963. Local muscle endurance with open and occluded intramuscular circulation. J. Physiol. 18: 804-807.

Van Wely, P., 1970. Design and disease. Appl. Ergon., 1: 262-269.

Weber, A., Jermini, C. and Grandjean, E.P., 1975. Relationship between objective and subjective assessment of experimentally induced fatigue. Ergonomics, 18(2): 151-156.

Wiker. S.F., 1986. Effects of relative hand location upon movement time and fatigue. Ph.D. Dissertation, The University of Michigan, Ann Arhor.

Wiker, S.F., Langolf, G.D. and Chaffin, D.B., 1989. Arm posture and human movement capability. Hum. Factors 31(4): 421-441.

Yamamoto, T. and Fujita, K., 1983. Postural effects on the aerobic capacity of forearm muscles. In: $\mathrm{H}$. Matsui and $\mathrm{K}$. Kobayashi (Eds.), Biomechanics VII-A. Human Kinetics Publishers, Champaign, IL, pp. 604-611.

Yata, H., Fukunaga, T., Matsuo, A.. Hyodo, K., Ryushi, T. and Asami, T., 1985. Effect of arm elevation on muscle circulation and exercise performance. In: D.A. Winter, R.W. Norman, R.P. Wells, K.C. Hayes and A.L. Patla (Eds.), Biomechanics IX-A. Human Kinetics Publishers, Champaign, IL. pp. 959-962. 